\title{
Environmental Effects in Galaxy Clusters: Infalling Groups in Abell 85 and 1367
}

\author{
H. Bravo-Alfaro, ${ }^{1}$ T. C. Scott,${ }^{2,3}$ E. Brinks, ${ }^{3}$ L. Cortese,${ }^{4}$ \\ P. Granados ${ }^{1}$ F. Navarro-Poupard,${ }^{1}$ Y. D. Mayya,${ }^{5}$ and F. Durret ${ }^{6}$ \\ ${ }^{1}$ Dept. de Astronomía, Universidad de Guanajuato, \\ Apdo. Postal 144, Guanajuato 36000, Mexico \\ email: hector@astro.ugto.mx \\ ${ }^{2}$ Instituto de Astrofísica de Andalucía, CSIC \\ Apdo. Correos 3004, E-18080 Granada, Spain \\ ${ }^{3}$ Centre for Astrophysics Research, Science \& Technology Research Institute \\ University of Hertfordshire, Hatfield AL10 9AB, UK \\ ${ }^{4}$ European Southern Observatory \\ Karl-Schwarzschild Str. 2, 85748 Garching bei Muenchen, Germany \\ ${ }^{5}$ Instituto Nacional de Astrofísica, Óptica y Electrńica, \\ Luis Enrique Erro 1, Tonantzintla 72840. Puebla, Mexico \\ ${ }^{6}$ Institut d'Astrophysique de Paris \\ 98 bis boulevard Arago, FR75014 Paris, France
}

\begin{abstract}
We are carrying out a multifrequency survey of late type galaxies in nearby clusters with the aim to investigate the effects exerted by both the very local and the global cluster environments. We report new VLA-HI images of galaxies in Abell 1367 and study the evolution of their gaseous component. In Abell 85 we perform a deep NIR imaging survey of the brightest spirals projected up to 1.0 Abell radius with the aim of unveiling possible gravitational effects on their stellar disks. Here we show preliminary results of these projects, mainly focused on infalling compact groups of galaxies moving towards their respective cluster centers.
\end{abstract}

Keywords. galaxies: clusters: individual (Abell 1367, Abell 85)

\section{Introduction}

Rich galaxy clusters are known to affect the evolution of their member galaxies. One of the strongest lines of evidence is the morphology-density relation, seen as the increase of the fraction of early-types as a function of galaxy density when we move from the outskirts to the cluster cores (Dressler 1980). In addition, many spirals are deficient in atomic hydrogen and display lower star formation compared with their field counterparts (e.g. Giovanelli \& Haynes 1985; Cayatte et al. 1990; Bravo-Alfaro et al. 2000; Gavazzi et al. 2006). Several physical mechanisms have been proposed to explain the different evolution between field and cluster spirals, classified in two big families; either we have hydrodynamical interactions of cluster galaxies with the hot intracluster medium (ICM) or gravitational interactions with nearby companions and/or with the cluster potential (Boselli \& Gavazzi 2006 and references therein.)

Furthermore, galaxies infalling into clusters happen to do so rather in groups than individually, making this issue even more complex. This would be in agreement with the hierarchical scenario for the formation of large-scale structures meaning that galaxy groups could be the natural preprocessing laboratories (Mihos 2004 and Fujita 2004). Several authors propose that preprocessing could represent the ideal scenario of formation 
of lenticulars following different kinds of mergers among spirals (e.g. Kodama \& Smail 2001). This issue is still a matter of debate, as nowadays very few infalling groups have been studied in detail. Probably the best known is the compact group of blue galaxies infalling to Abell 1367 (Sakai et al. 2002; Cortese et al. 2006). In this work we present preliminary results of HI and NIR surveys in two well studied clusters, Abell 1367 and Abell $85(\mathrm{z}=0.02$ and 0.05 respectively), where we detect groups of galaxies that are possibly infalling. In Section 2 we show the HI-distribution of two groups projected near the virial radius of A 1367, and in Sect. 3 we confirm the presence of a group near A 85 with some evidence of asymmetries in their stellar disks as seen in NIR imaging.

\section{The outskirts of Abell 1367: HI imaging}

Our VLA-HI imaging (D-Array) revealed two groups of galaxies, both projected within the range 1.5-2.0 degrees, NW from the centre of A 1367 ( 1.8-2.8 Mpc). Fig. 1(a) shows the HI distribution of the group RSCG 42 superposed on an SDSS r-band image. CGCG 097026/027 constitute the two optically dominant members. An HI tail emanates from CGCG 097-026, running NE along some $150 \mathrm{kpc}$. An asymmetry on CGCG 097-026's optical image is evident, indicating that tidal interactions are playing a role here. The HI tail extends from CGCG 097-026 until the blue galaxy SDSS J113709+200131, separated in projection by $100 \mathrm{kpc}$ and $\sim 55 \mathrm{~km} \mathrm{~s}^{-1}$ in relative velocity. Other low-mass members of the group (not shown in this figure) display offsets between the HI and optical positions, but it is not clear if interactions among the group members are the origin, or if it is an extension of a wider pattern of offsets previously reported by Scott et al. (2010).

Fig. 1(b) shows the HI distribution of the triple galaxy system, FGC 1287, CGCG 097$041 / 036$, within an optical velocity range of $50 \mathrm{~km} \mathrm{~s}^{-1}$. A striking tail, $\sim 250 \mathrm{kpc}$ long, originates from the Sdm spiral FGC 1287, with CGCG 097-041 projected SE and CGCG 097036 to the NW. The stellar disk of FGC 1287 is rather red $(g-i=1.22)$ and several features indicate tidal interactions and/or a minor merger among the group members. Strikingly,
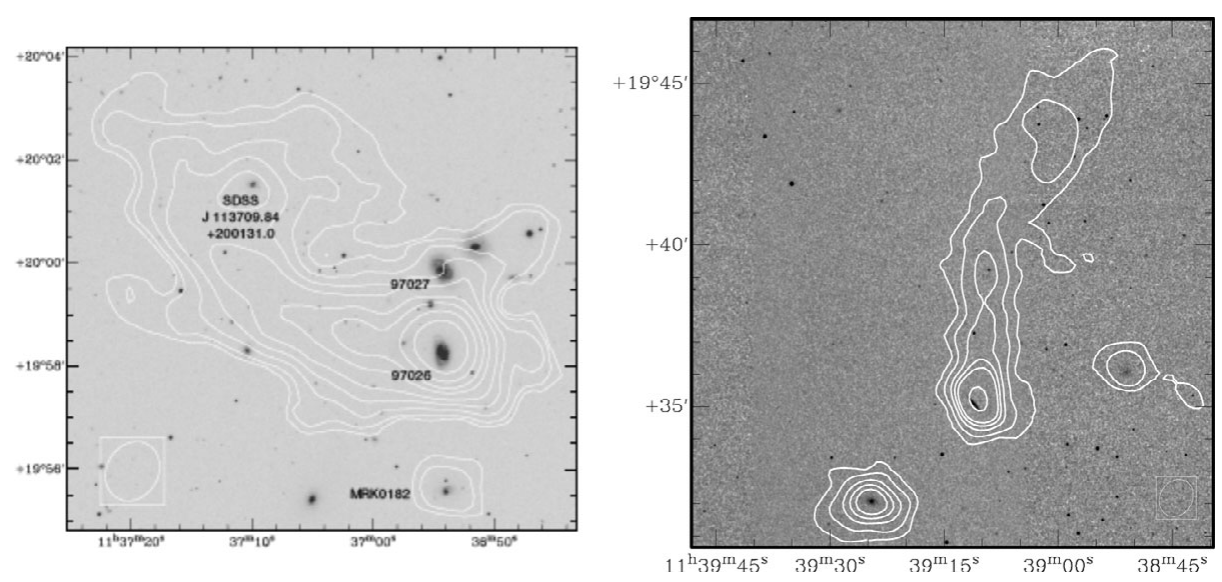

Figure 1. (a) Left panel: The HI distribution of the central region of the RSCG 42 group overlaid on an SDSS r-band image in gray scale. Contours come from a natural weighting HI

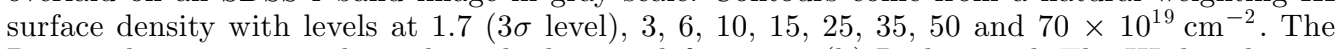
D-array beam size is indicated on the bottom left corner. (b) Right panel: The HI distribution of the FGC 1287 triplet overlaid on a 2MASS J-band image in gray scale. CGCG 097-036 is to the W and CGCG 097-041 is to the SE. Contours come from a natural weighting HI surface density with levels at 2.0 ( $3 \sigma$ level) $, 5,10,15,20$ and $40 \times 10^{19} \mathrm{~cm}^{-2}$. The D-array beam size is indicated on the bottom right corner. 


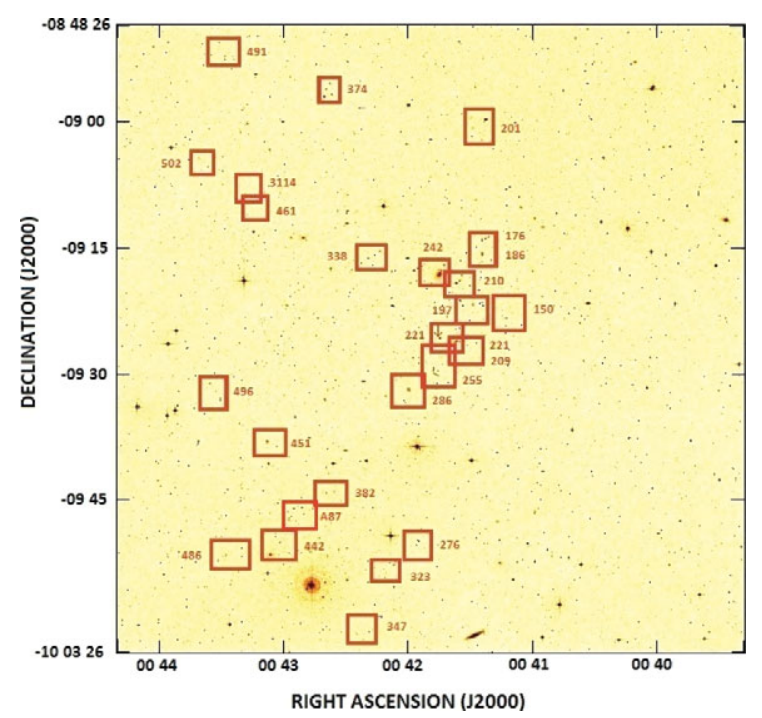

Figure 2. The position of the 25 fields imaged in the $J, H, K^{\prime}$-bands. Field numbers correspond to the dominant galaxy in the observed field following Durret et al. 1998.

the HI mass along the tail $\left(5.7 \times 10^{9} \mathrm{M} \odot\right)$ is larger than the HI within FGC 1287's disk $\left(3.7 \times 10^{9} \mathrm{M} \odot\right)$, and the velocity width $\left(\mathrm{W}_{20}\right)$ in the tail rises up to $388 \mathrm{~km} \mathrm{~s}^{-1}$, comparable to the disk, $453 \mathrm{~km} \mathrm{~s}^{-1}$. The tail velocity field (not shown in this paper) reveals an abnormal velocity structure, making its interpretation difficult. Very few spirals are known to display HI tails of projected length larger than $100 \mathrm{kpc}$, most of them interpreted as being produced by tidal interactions. The perturbation in these two systems, lack of alignment between the tails and A 1367's centre, and the fact that both groups are projected far from the high density zones of ICM, suggest that these tails are produced by tidal interactions within the group. A full analysis of both, this triplet and the group RSCG 42, will appear in a future paper (Scott et al. in prep).

\section{The brightest spirals of Abell 85: NIR imaging}

Fig. 2 draws the distribution of 25 fields in Abell 85 (each roughly $3^{\prime} \times 3^{\prime}$ ) obtained with the $2.1 \mathrm{~m}$ telescope (OAN, San Pedro Mártir, Mexico) and the NIR instrument CAMILA. The goal of this $J, H, K$ ' survey is to study the disk morphology of the (bright) spiral population looking for NIR asymmetries as indicators of tidal interactions. For several objects we will be able to correlate the stellar morphology with their HI content (van Gorkom et al. 2003; Bravo-Alfaro et al. 2009). We centered our NIR fields on galaxies brighter than $\mathrm{b}_{j}=18.0$ and bluer than $\mathrm{b}_{j}-\mathrm{r}_{f}=1.2$, projected within the $\sim 1$ Abell radius from the cluster center, and with velocities within A85 membership range: i.e. between 13,500 and 18,500 $\mathrm{km} \mathrm{s}^{-1}$ (Bravo-Alfaro et al. 2009). A preliminary analysis of the field labeled 176 in Fig. 2, projected $6^{\prime} \mathrm{NW}$ from the central cD galaxy, confirms the presence of a triplet of galaxies $\left(\Delta \mathrm{v} \sim 170 \mathrm{~km} \mathrm{~s}^{-1}\right)$ dominated by Abell0085:[DFL98]174, probably infalling with a high velocity relative to the cluster: their average velocity is $\sim 14,030 \mathrm{~km} \mathrm{~s}^{-1}$ compared with A85's systemic velocity of $16,600 \mathrm{~km} \mathrm{~s}^{-1}$.

The residual image obtained by subtracting an azimuthally symmetric component (constructed by fitting the isophotes with concentric ellipses) shows bar-like structures in the center of at least two galaxies. As an example, Fig. 3 shows the subtraction on the $\mathrm{K}^{\prime}$ image of this triplet, revealing important asymmetries. More details about the 


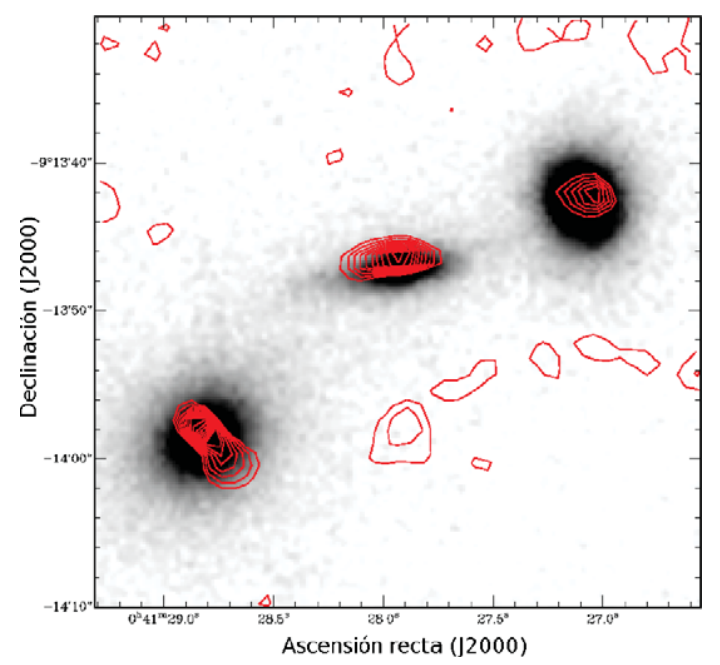

Figure 3. The residuals (contours) after subtraction of an axi-symmetric component from the K' image of a triplet in A 85, overlaid on our J-band image. Abell0085:[DFL98]174 is to the SE, [DFL98]167 is to the NW and [SDG98]1645 is in the middle. The cD galaxy is projected $6^{\prime} \mathrm{SE}$ from this field, and the relative velocity between the group and the cluster is larger than $2000 \mathrm{~km} \mathrm{~s}^{-1}$.

observations and the analysis of the whole sample will appear in a future paper (BravoAlfaro et al. in prep), where we will investigate how frequent it is that spiral galaxies suffer such tidal effects in a massive cluster like Abell 85.

\section{References}

Boselli, A. \& Gavazzi, G. 2006, PASP, 118, 517

Bravo-Alfaro, H., Cayatte, V., van Gorkom, J. H., \& Balkowski, C. 2000, AJ, 119, 580

Bravo-Alfaro, H., Caretta, C. A., Lobo, C., Durret, F., \& Scott, T. C. 2009, A\& A, 495, 379

Cortese, L., Gavazzi, G., Boselli, A., et al. 2006 A\&A, 453, 847

Dressler, A. 1980, ApJ, 236, 351

Durret, F., Felenbok, P., Lobo, C., \& Slezak, E. 1998, A\& AS, 129, 281

Fujita, Y. 2004, PASJ, 56, 29

Gavazzi, G., Boselli, A., \& Cortese, L., et al. 2006, A\&A, 446, 839

Giovanelli, R. \& Haynes, M. P. 1985, ApJ, 292, 404

Kodama, T. \& Smail, I. 2001, MNRAS, 326, 637

Mihos, J. C. 2004, in Clusters of Galaxies: Probes of Cosmological Structure and Galaxy Evolution, ed. J.S. Mulchaey, A. Dressler, and A. Oemler (N.Y. Cambridge, Univ. Press), 278

Scott, T. C., Bravo-Alfaro, H., \& Brinks, E. et al., 2010, MNRAS, 403, 1175

Sakai, S., Kennicutt, R. C., van der Hulst, J. M., \& Moss, C. 2002, ApJ, 578, 842

van Gorkom, J. H., Bravo-Alfaro, H., \& Dwarakanath, K. S., et al. 2003, ApSS, 285, 219 